\title{
Giant super-poissonian shot noise in spin-polarized SET structures
}

\author{
L.Y. Gorelik ${ }^{1}$, S.I. Kulinich ${ }^{2,3}$, R.I. Shekhter ${ }^{2}$, M. Jonson ${ }^{2}$, and V.M. Vinokur ${ }^{4}$ \\ ${ }^{1}$ Department of Applied Physics, Chalmers University of Technology, SE-412 96 Göteborg, Sweden \\ E-mail: gorelik@fy.chalmers.se \\ ${ }^{2}$ Department of Physics, Göteborg University, SE-412 96 Göteborg, Sweden \\ ${ }^{3}$ B.I. Verkin Institute for Low Temperature Physics and Engineering, 47 Lenin Ave., 61103 Kharkov, Ukraine \\ ${ }^{4}$ Materials Science Division, Argonne National Laboratory, 9700 South Cass Av, Argonne, Illinois 60439, USA
}

Received January 15, 2007

\begin{abstract}
We study transport of spin-polarized electrons through a magnetic single-electron transistor (SET) in the presence of an external magnetic field. Assuming the SET to have a nanometer-sized central island with a single electron level, we find that the zero-frequency shot noise diverges as the on-dot spin-flip rate goes to zero, provided the source and drain leads are completely polarized in the same direction. We present an analytical expression for the low-frequency super-poissonian shot noise that allows one to specify the necessary conditions for the experimental observation of the phenomenon.
\end{abstract}

PACS: 73.23.-b Electronic transport in mesoscopic systems;

73.40.Gk Tunneling (for tunneling in quantum Hall effects).

Keywords: single-electron transistor, spin-flip time, shot-noise, Coulumb blockade, spin-polarized electrons.

Confinement of electrons to a quantum of dot nanometer size crucially reduces the spin relaxation frequency [1] and allows for the implementation of various schemes where quantum information is carried by the spin of electrons [2-5]. Experimental evidence that the spin-flip time in quantum dots can reach the millisecond range has recently been obtained* $[6,8]$. Such long spin relaxation times is obviously an important prerequisite for spintronics-based quantum information processing. At the same time, the advantage coming from the extremely weak spin relaxation might obviously be an obstacle for its experimental study, demanding measurements of extremely high accuracy and sensitivity [6]. This is why any phenomenon that is exceedingly sensitive exclusively to spin relaxation should be of considerable practical importance. The Zeeman splitting of energy levels is the phenomenon traditionally invoked to discriminate between contributions from different electronic spin-states to observable physical properties. Today, a number of different methods are used to create nonequilibrium spin populations and to study how they relax towards equilibrium. Transport measurements, which usually provide an excellent tool for such studies, are limited when it comes to observing very weak spin relaxation by the necessity to measure the extremely small electrical currents that result from the electrons being trapped for long times on the dot [6]. A large sensitivity to slow spin relaxation rates therefore requires a careful choice of observable. Recently, numerical simulations have revealed that shot noise in single-electron transistor (SET) structures, which significantly increases with the spin polarization of the tunneling electrons $[7,9]$, could be such an observable. In this paper we present an analytical study of this phenomenon where we show that spin-dependent tunneling in combination with the Coulomb

* The effect of intradot spin-flip scattering on shot noise was recently studied in Ref. 7. An increased shot-noise was reported for tunneling of polarized electrons. However, this effect was analyzed only numerically, which does not allow the results to be used as a tool for spectroscopy. 
blockade phenomenon in SETs results in shot noise characterized by a giant super-poissonian Fano factor at low frequencies. Consequently low frequency noise measurements provides a tool for detecting slow spin flip rates in a quantum dot.

Below we will study the transport of spin polarized electrons through a quantum using dot the SET geometry represented in Fig. 1. Two magnetic leads, biased by a voltage $V$ serve as source and drain for the spin polarized electrons, while two electronic states with different spin orientation are available on the dot. An external magnetic field oriented perpendicular to the magnetization of the leads (which we assume are parallel) induces coherent spin-flip processes on the dot.

To study the electronic transport through this system we will use the Hamiltonian $\hat{\mathcal{H}}$, where

$$
\hat{\mathcal{H}}=\hat{\mathcal{H}}_{l}+\hat{\mathcal{H}}_{d}+\hat{\mathcal{H}}_{T}+\hat{\mathcal{H}}_{\text {env }}
$$

and

$$
\begin{gathered}
\hat{\mathcal{H}}_{l}=\sum_{\alpha, \sigma, \kappa} \varepsilon_{\alpha, \sigma, \kappa} a_{\alpha, \sigma, \kappa}^{\dagger} a_{\alpha, \sigma, \kappa} \\
\hat{\mathcal{H}}_{d}=\sum_{\sigma} \varepsilon a_{\sigma}^{\dagger} a_{\sigma}-\frac{U}{2} a_{\uparrow}^{\dagger} a_{\downarrow}^{\dagger} a_{\uparrow} a_{\downarrow}-\mu \sum_{i, \sigma, \sigma^{\prime}} H_{i} a_{\sigma}^{\dagger} \tau_{i}^{\sigma, \sigma^{\prime}} a_{\sigma^{\prime}} \\
\mathcal{H}_{T}=\sum_{\alpha, \sigma, \kappa} T_{\kappa}\left(a_{\alpha, \sigma, \kappa}^{\dagger} a_{\sigma}+\text { H.c. }\right)
\end{gathered}
$$

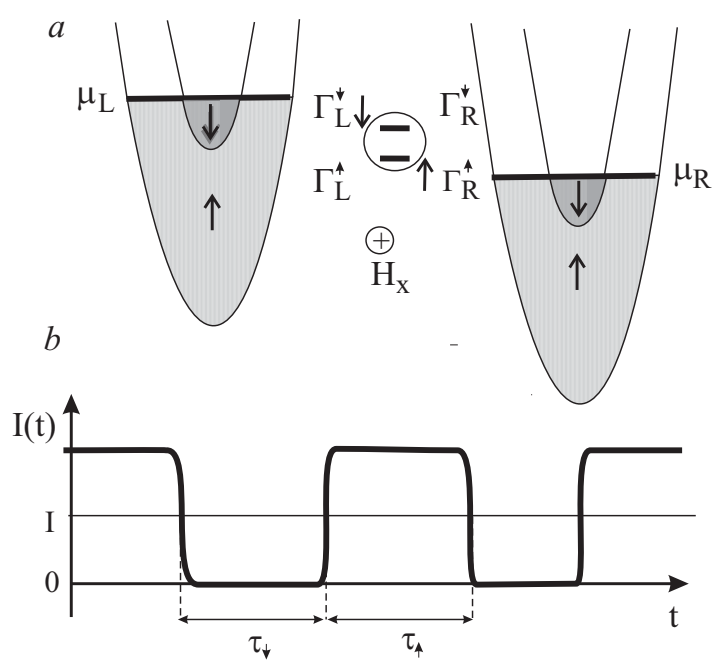

Fig. 1. $a-$ Sketch of the nano-magnetic SET device discussed in the text: a quantum dot, modelled as a single spin-degenerate electron level, is coupled to two leads with parallel magnetization. The tunneling rates $\Gamma_{L(R)}^{\uparrow(\downarrow)}$ describe the coupling between and dot electrodes, while the external magnetic field $H_{x}$ induces flips between the spin-up and the spin-down state on the dot. The difference $\mu_{L}-\mu_{R}=e V$ in electrochemical potential of the leads is due to a bias voltage $V . b-$ Schematic picture of the current fluctuations in the case when the leads are completely polarized in the same direction ( $I$ is the average current).
Here $\hat{\mathcal{H}}_{l}$ describes noninteracting electrons in the leads and $a_{\alpha, \sigma, \kappa}^{\dagger}\left(a_{\alpha, \sigma, \kappa}\right)$ is the creation (annihilation) operator for electrons in lead $\kappa=(L, R)$ with energy $\varepsilon_{\alpha, \sigma, \kappa}$ and spin projection $\sigma=(\uparrow, \downarrow)$. The electron density of states $v_{\kappa}^{\sigma}$ in each lead is assumed to be independent of energy but strongly dependent on spin direction. The electrons in each lead are held at a constant electrochemical potential $\mu_{L, R}=E_{F} \mp e V / 2$, where $e$ is the charge of an electron, $V>0$ is the bias voltage, and $E_{F}$ is the Fermi energy of the ferromagnetic metal. The Hamiltonian $\hat{\mathcal{H}}_{d}$ describes electronic states in the dot and their coupling to the external magnetic field $\mathbf{H}=\left(H_{x}, 0, H_{z}\right) ; \mu$ is the Bohr magneton and intra-dot electron correlations are characterized by the Coulomb energy $U$; the operator $a_{\sigma}^{\dagger}\left(a_{\sigma}\right)$ creates (destroys) an electron with spin $\sigma$ and $\tau_{i}^{\sigma, \sigma^{\prime}}$ are Pauli matrix elements $(i=x, y, z)$. The third term in (1) represents spin-conserving tunneling of electrons between dot and leads while the last term describes the interaction between dot-electrons and the magnetic environment.

In this paper we will study the case when the bias voltage $V$ is much smaller than both the spacing between spatially quantized levels on the dot and the Coulomb energy $U$, but much larger than the Zeeman energy $\Delta=\mu \mathrm{H}_{z}$ (see Fig. 1,a). This means that only one electron may be accumulated on the dot. In addition, we will focus on the situation when the electrons on the leads are almost completely polarized in the same direction (for definiteness in the «up» direction): $\varepsilon_{\mathrm{\kappa}}=v_{\mathrm{K}}^{\downarrow} / \nu_{\mathrm{K}}^{\uparrow}=\Gamma_{\mathrm{K}}^{\downarrow} / \Gamma_{\mathrm{\kappa}}^{\uparrow}<<1\left(\Gamma_{\mathrm{\kappa}}^{\sigma}=\right.$ $\left.=2 \pi \hbar^{-1} v_{\kappa}^{\sigma} T_{\kappa}^{2}\right)$.

The two phenomena of electron spin-polarization in the leads and Coulomb blockade of double electron occupancy on the dot determine the current that flows through the system. To understand qualitatively the peculiarities of electronic transport in this situation we consider the limiting case when the electrons are completely polarized $\left(\varepsilon_{L}=\varepsilon_{R}=0\right)$. In this case a transition of the spin of the dot electron from «up» to «down» traps the electron on the dot, and prevents a current to flow through the system (since the Coulomb blockade prevents another, spin-up, electron to enter the dot). This is why the temporal variation of the current strictly follows the switching of electronic spin, allowing for such events to be counted. A schematic picture of the current fluctuations $\delta \mathbf{I}(t) \equiv \mathbf{I}(t)-\mathbf{I}$, where $\mathbf{I}$ is the average value of the current, is shown in Fig. 1, $b$. It is important that the current-current correlation function $S(0)=$ $=\int d t\langle\langle\delta \hat{\mathbf{I}}(t) \delta \hat{\mathbf{I}}(0)\rangle\rangle$, where $\langle\langle\ldots\rangle\rangle$ denotes a statistical average, serves as a measure of the time $\tau_{\downarrow}$ during which the electron is trapped on the dot in the spin-down state - the state that prevents a current to flow through the system. Two possible channels for the escape of the electron from this state should be identified: a) a stochastic spin-flip process caused by the interaction with the environment and characterized by the spin relaxation time $\tau_{\text {rel }}=\gamma_{1}^{-1}$ and b) a magnetic-field induced coherent transition to the spin-up 
state. The latter is characterized by the rate $v_{H}=\left(\mu H_{x}\right)^{2} \mathcal{N}(\Delta / 2) / \hbar$, w $h$ e $\quad$ r $\quad$ e $\mathcal{N}(\varepsilon)=(2 / \pi) \times$ $\times \operatorname{Im}\left(2 \varepsilon+\Delta-i \Gamma_{R}^{\uparrow}\right)^{-1}$ is the density of spin-up states on the dot. Therefore $\tau=\left(\gamma_{1}+v_{H}\right)^{-1}$ and $S(0)$ may be estimated as

$$
S(0) \propto\left(\gamma_{1}+v_{H}\right)^{-1} .
$$

If $H_{x}=0$ we have $v_{H}=0$ and one can see from the above expression that the zero-frequency shot noise is a divergent function of the spin relaxation rate reaching giant values for slow enough relaxation. Below we will show that the average current, on the other hand, does not at all depend on the external magnetic field and the internal spin relaxation rate if $\varepsilon_{\uparrow}=\varepsilon_{\downarrow}=0$. Hence a simultaneous measurement of both the current and the noise for opposite polarities of the bias voltage allows one to deduce the value of the spin-flip rate.

In order to quantitatively investigate the current and shot noise in the SET structure described above one can use the quantum master equation for the reduced density matrix $\hat{\rho}_{r}(t)=\operatorname{Tr}_{l} \hat{\rho}(t)$ that describes the state of the $\operatorname{dot}(\hat{\rho}$ is the total density matrix and $\operatorname{Tr}_{l}$ denotes a trace over all electronic states in the leads).

The operator $\hat{\rho}_{r}(t)$ acts on the Fock space of the quantum dot, which in the Coulomb blockade regime is spanned by the three basis vectors $|0\rangle,|\uparrow\rangle \equiv a_{\uparrow}^{\dagger}|0\rangle,|\downarrow\rangle \equiv a_{\downarrow}^{\dagger}|0\rangle$. In this basis $\hat{\rho}_{r}(t)$ is a $3 \times 3$ matrix. The diagonal element $\left\langle 0\left|\hat{\rho}_{r}(t)\right| 0\right\rangle$ represents the probability for the dot to be unoccupied, the singly occupied dot is described by the $2 \times 2$ matrix block $\hat{\rho} \equiv\left(\hat{\rho}_{r}\right)_{\sigma, \sigma^{\prime}} \equiv\left\langle\sigma\left|\hat{\rho}_{r}\right| \sigma^{\prime}\right\rangle$, while nondiagonal elements $\left\langle\sigma\left|\hat{\rho}_{r}\right| 0\right\rangle$ equal zero.

The time evolution of the probability $\rho$ and the density matrix $\hat{\rho}$ is determined by a system of equations that describe the coupled processes of stochastic charge transfer and coherent spin dynamics [10] (below we will take $e=\hbar=1)$,

$$
\frac{\partial \hat{\rho}}{\partial t}=-\operatorname{Tr}\left\{\hat{\Gamma}_{L}\right\} \rho+\operatorname{Tr}\left\{\hat{\Gamma}_{R} \hat{\rho}\right\},
$$

$$
\frac{\partial \hat{\rho}}{\partial t}=i\left[\frac{\Delta \hat{\tau}_{z}}{2}+\mu H_{x} \hat{\tau}_{x}, \hat{\rho}\right]+\hat{\Gamma}_{L} \rho-\frac{1}{2}\left\{\hat{\Gamma}_{R}, \hat{\rho}\right\}+\mathcal{L}_{\gamma}\{\hat{\rho}\}
$$

where $\hat{\Gamma}_{\kappa}=\left(\Gamma_{\kappa}^{\uparrow}+\Gamma_{\kappa}^{\downarrow}\right) \hat{I} / 2+\left(\Gamma_{\kappa}^{\uparrow}-\Gamma_{\kappa}^{\downarrow}\right) \tau_{z} / 2$. The linear operator $\mathcal{L}_{\gamma}\{\hat{\rho}\}$ describes the spin relaxation in a singly occupied dot caused by interaction with the environment. In the general case it can be written in the form

$$
\mathcal{L}_{\gamma}\{\hat{\rho}\}=-\gamma_{1} \hat{\tau}_{z}\left[\operatorname{Tr}\left(\hat{\tau}_{\hat{z}} \hat{\rho}\right)-\xi \operatorname{Tr} \hat{\rho}\right]-\gamma_{2} \sum_{i \neq z} \tau_{i} \operatorname{Tr}\left(\hat{\tau}_{i} \hat{\rho}\right) .
$$

Here $\gamma_{1}$ and $\gamma_{2}$ are, respectively, the spin relaxation rate and the spin dephasing rate, while the coefficient $\xi=$ $=\tanh (\beta \Delta / 2)$ ( $\beta$ is the inverse temperature) characterizes the difference in the populations of spin-up and spindown states if the system is in equilibrium with the environment. Here we will consider the case when the thermal energy is much higher than the Zeeman splitting (but lower than the bias voltage) and take $\xi=0$. The spin relaxation rate $\gamma_{1}$ defines the rate of direct spin-flip transitions induced by the environment and as a result has a first-hand influence on the low-frequency shot noise. In contrast, the dephasing rate $\gamma_{2}$, which controls the decay of nondiagonal elements of the reduced density matrix, affects spin-flip processes only indirectly. As we will see below, it only renormalizes the effect of the external magnetic field.

Within our approach the average current, $I=\langle\langle\hat{I}\rangle\rangle=$ $=i\left[\hat{H}, \hat{N}_{R}\right]$, where $\hat{N}_{R}$ is the electron number operator in the right lead, can be calculated from the formula [10]

$$
I=\operatorname{Tr}\left\{\hat{\Gamma}_{R} \hat{\rho}_{0}\right\},
$$

where $\left(\rho_{0}, \hat{\rho}_{0}\right)$ represent the stationary solution of Eq. (3). In our further considerations we will use the formalism developed in Ref. 11. Following this approach it is convenient to rewrite Eqs. (5) in the form

$$
\frac{\partial|\chi\rangle}{\partial t}=\mathbf{L}|\chi\rangle
$$

where the column vector $|\chi\rangle$ is given by $|\chi\rangle=$ $=\left(\rho, \rho_{\uparrow \uparrow}, \rho_{\downarrow \downarrow}, \rho_{\uparrow \downarrow}, \rho_{\downarrow \uparrow}\right)^{T}=\left\langle\left.\chi\right|^{T}\right.$, while the matrix $\mathbf{L}$ is determined by Eqs. (3). In this notation the formula for the average current, Eq. (5), takes the form

$$
I=\left\langle\chi_{0}^{T}|\mathbf{J}| \chi_{0}\right\rangle,
$$

were $\left|\chi_{0}\right\rangle$ obeys equation

$$
\mathbf{L}\left|\chi_{0}\right\rangle=0 \text {. }
$$

By this means the elements of the steady-state density matrix, the vector $\left|\chi_{0}^{T}\right\rangle=(1,1,1,0,0)^{T}$, satisfy the equation $\mathbf{L}^{T}\left|\chi_{0}^{T}\right\rangle=0$, and $\mathbf{J}$ is a «current» matrix that has only two nonzero elements $\hat{J}_{12}=\Gamma_{R}^{\uparrow}, \hat{J}_{13}=\Gamma_{R}^{\downarrow}$. We solved Eq. (8) and found the following expression for the average current when $\varepsilon_{\mathrm{\kappa}}<<1$ :

$$
I=I_{0}\left(\frac{h^{2}+\varepsilon_{R}+\tilde{\gamma}_{1}}{h^{2}+\varepsilon_{R}+\eta\left(\varepsilon_{L}-\varepsilon_{R}\right)+\tilde{\gamma}_{1}}+O\left(\varepsilon_{\kappa}\right)\right) .
$$

Here $\widetilde{\gamma}_{1}=\gamma_{1} / \Gamma_{R}^{\uparrow}, I_{0}=\Gamma_{R}^{\uparrow} \eta$ and

$$
I_{0}=\frac{\Gamma_{L}^{\uparrow} \Gamma_{R}^{\uparrow}}{2 \Gamma_{L}^{\uparrow}+\Gamma_{R}^{\uparrow}}, h^{2}=\left(\frac{H_{x}}{H_{0}}\right)^{2}=\frac{\left(\mu H_{x}\right)^{2}}{\Delta^{2}+\left(\Gamma_{R}^{\uparrow} / 2+2 \gamma_{2}\right)^{2}} .
$$

Analyzing the above expression one finds that in the absence of spin-flip processes $\left(H_{x}=0, \gamma_{1}=0\right)$ the current vanishes if $\varepsilon_{R} \rightarrow 0$. Indeed, under such conditions the electron can not leave the spin-down state and the current is blocked forever. Then, from (9) it follows that with identical leads $\left(\varepsilon_{L}=\varepsilon_{R} \equiv \varepsilon\right)$ the current is equal to $I_{0}+O(\varepsilon)$ and does not depend (to leading order in $\varepsilon$ on the 
magnetic field, relaxation rates or Zeeman splitting. Below we will study the shot noise for this very case.

The noise power spectrum is defined as twice the Fourier transform of the current-current correlation function (see Ref. 12 for a review):

$$
S(\omega)=2 \int d t \mathrm{e}^{i \omega t}\left[\langle\langle\hat{I}(t) \hat{I}(0)\rangle\rangle-I^{2}\right] .
$$

Within our formalism the correlation function $\langle\langle\hat{I}(\mathrm{t}) \hat{I}(0)\rangle\rangle$ can be expressed in the form [11]

$$
\begin{gathered}
\langle\langle\hat{I}(t) \hat{I}(0)\rangle\rangle=\operatorname{I} \delta(t)+\theta(t)\left\langle\chi_{0}^{T}|\mathbf{J P}(t) \mathbf{J}| \chi_{0}\right\rangle+ \\
+\theta(-t)\left\langle\chi_{0}^{T}|\mathbf{J P}(-t) \mathbf{J}| \chi_{0}\right\rangle,
\end{gathered}
$$

where $\mathbf{P}(t)=\exp \mathbf{L} t$ is an evolution operator. The first term on the right hand side of Eq. (11) is the self-correlation term.

Using Eqs. (10) and (11) one can rewrite the formula for the shot noise in terms of the eigenvalues $\lambda_{i}$ of the matrices $\mathbf{L}, \mathbf{L}^{T}$ and their associated eigenvectors $\left|\lambda_{i}\right\rangle$, $\left|\lambda_{i}^{T}\right\rangle$. Taking into account that the eigenvalue, $\lambda_{0}$ say, that corresponds to the steady-state solution of Eq. (8), is zero (accordingly $\left|\lambda_{0}\right\rangle \equiv\left|\chi_{0}\right\rangle,\left|\lambda_{0}^{T}\right\rangle=\left|\chi_{0}^{T}\right\rangle$ ), we get

$$
\begin{aligned}
& S(\omega)=2 I-\sum_{i \neq 0} \frac{4 \lambda_{i}}{\omega^{2}+\lambda_{i}^{2}} T\left(\lambda_{0}, \lambda_{i}\right), \\
& T\left(\lambda_{0}, \lambda_{i}\right)=\frac{\left\langle\lambda_{0}|\mathbf{J}| \lambda_{i}\right\rangle\left\langle\lambda_{i}|\mathbf{J}| \lambda_{0}\right\rangle}{\left\langle\lambda_{i} \mid \lambda_{i}\right\rangle} .
\end{aligned}
$$

The eigenvalues $\lambda_{i}$ are given by zeros of the characteristic polynomial $P(\lambda) \equiv \operatorname{Det}\|\mathbf{L}-\lambda \mathbf{I}\|=\lambda P_{0}(\lambda)$. We have found that in the general case (with arbitrary values of the parameters $\varepsilon, \gamma_{i}, H_{x}$ and $\Delta$ ) the polynomial $P_{0}(\lambda)$ has the form

$$
\begin{gathered}
P_{0}(\lambda)=\left[\left(\lambda+\Gamma_{R}+2 \gamma_{2}\right)^{2}+\Delta^{2}\right] \times \\
\times\left[\lambda^{2}+2 \lambda\left(\Gamma_{L}+\Gamma_{R}+\gamma_{1}\right)+D\right]+ \\
+4\left(\mu H_{x}\right)^{2}\left(\lambda+\Gamma_{R}+2 \gamma_{1}\right)\left(\lambda+2 \Gamma_{L}+\Gamma_{R}\right),
\end{gathered}
$$

where

$$
D=\left(2 \Gamma_{L}^{\uparrow}+\Gamma_{R}^{\uparrow}\right)\left(\varepsilon \Gamma_{R}^{\uparrow}+\gamma_{1}+\varepsilon \gamma_{1}\right) \text { and } \Gamma_{\mathrm{K}}=\Gamma_{\mathrm{K}}^{\uparrow}(1+\varepsilon) / 2 \text {. }
$$

From the above expression it follows that one of the roots of the characteristic polynomial $\lambda_{1}$ vanishes like

$$
\lambda_{1} \simeq-A \Gamma_{R}^{\uparrow}\left(h^{2}+\varepsilon+\widetilde{\gamma}_{1}\right)
$$

if $\varepsilon, \gamma_{1}$ and $H_{x}$ go to zero;

$$
A=\left(2 \Gamma_{L}^{\uparrow}+\Gamma_{R}^{\uparrow}\right) /\left(\Gamma_{L}^{\uparrow}+\Gamma_{R}^{\uparrow}\right) \in(1,2)
$$

is a dimensionless coefficient of order one.

As a consequence, the correlation function $S(0)$ may according to Eq. (12) diverge and exhibit a giant superpoissonian behavior for small values of these parameters. By calculating the «transition» coefficient $T\left(\lambda_{0}, \lambda_{1}\right)$, in the case of strongly polarized leads $(\varepsilon<<1)$ and small spin-flip frequency

$$
v_{s f}=\Gamma_{R}^{\uparrow} h^{2}+\gamma_{1}<<\Gamma_{R}^{\uparrow}
$$

we get (to leading order) the following expression for the low-frequency Fano factor $F(\omega)=S(\omega) / 2 I^{*}$

$$
F(\omega) \simeq 2 \eta^{2} A^{2} \Gamma_{R}^{\uparrow} \frac{v_{s f}+\varepsilon \Gamma_{R}^{\uparrow}}{\omega^{2}+A^{2}\left(v_{s f}+\varepsilon \Gamma_{R}^{\uparrow}\right)^{2}} .
$$

Equation (16), together with (9) and (15), is the main result of our considerations. First, from these equations it follows that the spin dephasing rate $\gamma_{2}$, in contrast to the spin relaxation rate $\gamma_{1}$, only influences the contribution of the transverse magnetic field to the spin-flip process, and that its effect, according to Eq. (9), is strongly restricted by the condition $4 \gamma_{1} \geq \Gamma_{R}^{\uparrow}$. Then from (16) one can see that the zero-frequency shot noise diverges as $1 / v_{s f}$ demonstrating a high sensitivity to the intensity of spin-flip processes, if the leads are completely polarized $(\varepsilon=0)$. If the polarization of the leads is not complete, the spin-flip processes significantly affect the shot noise only if the spin-flip frequency $v_{s f}$ is not much smaller than the «minority» tunneling rate $\Gamma_{R}^{\downarrow}$ :

$$
\Gamma_{R}^{\uparrow}\left(H_{x} / H_{0}\right)^{2}+\gamma_{1}>\varepsilon \Gamma_{R}^{\uparrow} \text {. }
$$

This brings an important restriction on the use of shot noise measurements as a tool for probing spin-flip processes. Indeed, from the above inequality it follows that in order to increase the sensitivity of shot noise to spin flips (in particular to the spin relaxation rate $\gamma_{1}$ ) one has to diminish either $\varepsilon$ (by increasing the polarization in the leads) or the «majority» tunneling rate $\Gamma_{R}^{\uparrow}$. However, the latter alternative is not preferable, since it decreases the value of the current.

If there is no transverse magnetic field the spin-flip frequency coincides with the spin-relaxation rate $\gamma_{1}$. For this case we have obtained the following expression for $F(\omega)$ :

$$
F(\omega)=1-\frac{\alpha_{1} \omega^{2}+\alpha_{2}}{\omega^{4}+\alpha_{3} \omega^{2}+D^{2}},
$$

* Equation (9) shows that the average current does not depend (in leading order) on the spin-flip rate $v_{S f}$ if the leads are strongly polarized. Hence the low-frequency Fano factor completely characterizes the behavior of the low-frequency shot noise. 
where

$$
\begin{gathered}
\alpha_{1}=2\left(\Gamma_{L}^{\uparrow} \Gamma_{R}^{\uparrow}+\Gamma_{L}^{\downarrow} \Gamma_{R}^{\downarrow}\right), \\
\alpha_{2}=8 \Gamma_{L}\left(\Gamma_{L}+\Gamma_{R}+\gamma_{1}\right)\left(\Gamma_{R}^{\uparrow} \Gamma_{R}^{\downarrow}+2 \gamma \Gamma_{R}\right)-\alpha_{1} D, \\
\alpha_{3}=4\left(\Gamma_{L}+\Gamma_{R}+\gamma_{1}\right)^{2}-2 D,
\end{gathered}
$$

which is valid for any values of $\omega, \Gamma_{\mathrm{K}}^{\sigma}$, and $\gamma_{1}$. One can easily check that this expression is in agreement with Eq. (16). From Eq. (17), as well from Eq. (16), it follows that the zero-frequency Fano factor diverges as $1 / \gamma_{1}$ when the relaxation rate goes to zero if $\varepsilon, H_{x}=0$, i.e., if the leads are completely polarized and the transverse magnetic field is zero. On the other hand, if the leads are fully polarized and spin-flip processes are absent $\left(\gamma_{1}=0\right)$, the Fano factor $F(0)$ should coincide with the one that has been calculated for spinless electrons [11]. The correct value of the shot noise at zero frequency may be obtained from Eq. (17) if one takes the appropriate limits in the correct order, so that

$$
F\left(\omega=0, \gamma_{1}=0\right)=\lim _{\omega \rightarrow 0} \lim _{\gamma_{1} \rightarrow 0} F\left(\omega, \gamma_{1}\right) .
$$

To understand this ordering one has to take into account that an infinite time is needed to measure zero-frequency noise, while in a real experimental situation the frequency under consideration can not be smaller than the inverse observation time.

We have also calculated the zero-frequency shot noise in the limit of a strong transverse magnetic field, $\chi H_{x}^{2}>>1$. It turns out that only one term in the sum (12) gives a nonvanishing contribution to the shot-noise in this limit; the corresponding root of the characteristic polynomial (13) has the asymptotic value

$$
\lambda_{h}=-\left(2 \Gamma_{L}+\Gamma_{R}\right)+O\left(1 / \chi H_{x}^{2}\right) .
$$

Calculating the «transition» coefficient $T\left(\lambda_{0}, \lambda_{h}\right)$ we obtained the following expression for the zero-frequency Fano factor:

$$
F(0) \simeq 1-\frac{4 \Gamma_{L} \Gamma_{R}}{\left(2 \Gamma_{L}+\Gamma_{R}\right)^{2}} .
$$

From this expression one can see that in the limit of a strong magnetic field the zero-frequency shot noise, like the average current, does not depend on the spin-flip rate, in particular not the rate due to the magnetic field.
In conclusion, we have studied transport of spin-polarized electrons through a magnetic SET in the presence of an external magnetic field. Assuming the SET to have a nanometer-sized central island with a single-electron level we found that the zero-frequency shot noise diverges as the rate for spin-flips on the goes dot to zero if leads are completely polarized in the same direction. We presented an analytical expression for the low-frequency super-poissonian shot noise and discussed the conditions required for the experimental observation of such an enhancement of the low-frequency noise.

This work was supported in part by the European Commission through project FP6-003673 CANEL of the IST Priority. The views expressed in this publication are those of the authors and do not necessarily reflect the official European Commission's view on the subject. Financial support from the Swedish SSF, the Swedish VR, and from the U.S. DOE Office of Science through contract No. W-31-109-ENG-38 is also gratefully acknowledged.

1. V. Khaetskii and Yu.V. Nazarov, Phys. Rev. B61, 12639 (2000).

2. D.D. Awschalom, D. Loss, and N. Samarth, Semiconductor Spintronics and Quantum Computation, Springer, Berlin (2002).

3. I. Zutic, J. Fabian, and S. Das Sarma, Rev. Mod. Phys. 76, 323 (2004).

4. M.A. Nielsen and I.L. Chuang, Quantum Computation and Quantum Information, Cambridge University Press, New York (2000).

5. V. Cerletti, W.A. Coish, O. Gywat, and D. Loss, Nanotechnology 16, R27 (2005).

6. R. Hanson, B. Witkamp, L.M. Vandersypen, L.H. Willems van Beveren, J.M. Elzerman, and L.P. Kouwenhoven, Phys. Rev. Lett. 91, 196802 (2003).

7. I. Djuric, B. Dong, and H.L. Cui, J. Appl. Phys. 99, 063710 (2006); Appl. Phys. Lett. 87, 032195 (2005).

8. J.M. Elzerman, R. Hanson, L.H.W. van Beveren, B. Witkamp, L.M.K. Vandersypen, and L.P. Kouwenhoven, Nature (London) 430, 431 (2004).

9. Collosal Magnetoresistive Oxides, Y. Tokura (ed.), Gordon and Breach, Amsterdam (2000).

10. L.Y. Gorelik, Phys. Rev. Lett. 95, 116806 (2005).

11. Z. Hershfield, Phys. Rev. B47, 1967 (1993).

12. M.J.M. de Jong and C.W.J. Beenakker, in Mesoscopic Electron Transport, L.L. Sohn, G. Schön, and L.P. Kouwenhoven (eds.), Kluwer Academic Publisher, Dordrecht (1997). 